\title{
Industry 4.0 and basic principles of a new architecture for control of power plants processes
}

\author{
Nikolay Amosov ${ }^{1}$, Alexander Andryushin ${ }^{2}$, Edik Arakelyan², and Anatoliy Kosoy ${ }^{2}$ \\ ${ }^{1}$ Peter the Great St. Petersburg Polytechnic University, 195251, Politechnicheskaya st., 29, Russian \\ Federation \\ ${ }^{2}$ National Research University "MPEI", Thermal and Atomic Power Engineering Institute, 111250, \\ Krasnokazarmennaya st., 14, Russian Federation
}

\begin{abstract}
The results of the level analysis of intellectuality and efficiency of up-to-date automated process control systems (APCS) based on software and hardware systems (SHS) are presented. It was demonstrated that, despite the widespread implementation of modern software and hardware systems during the construction of new APCS and upgrading the existing ones for thermal power plants (TPP), improvement of the process control quality, optimization of their modes and parameters take place generally at the equipment and power unit level and to a far lesser extent - at the power plant level and as a result - insufficient level of automation and low technical and economic efficiency. Another conclusion from the performed analysis when implementing control algorithms in the APCS based on the up-to-date SHS, their wide data and software capabilities are not fully used. The main ways of further APCS improvement for the purpose of further increasing of their efficiency and the level of intelligence of SHS based APCS of power plants are presented. The possibility of their implementation is considered with application of the basic principles laid in the concept Industry 4.0. The possible economic effect from the implementation of the proposed solutions for increasing the intelligence and efficiency of the SHS based APCS was assessed. A brief description of the configuration of the proposed SHS based control system is given.
\end{abstract}

The technological process of up-to-date TPP differs by the complexity of the interrelationships between a large number of equipment, high parameters of the working environment, stringent requirements for the accuracy of their regulation, and this has led to the constant search and implementation of new methods for improving the control systems based on the new automatic control hardware. The analysis shows that the current stage of power plants APCS development is moving in two main directions [1,2]:

- Improvement of the automation equipment itself, first of all, software and hardware system (SHS), on the basis of which new (conventional) APCS are created and the existing ones are upgraded;

*Corresponding author: edik_arakelyan@inbox.ru 
- Increase in the processes automation level, providing a significant improvement in the quality and efficiency for maintenance of technological modes of equipment both in the range of workloads, and in the processes of equipment start and stop.

Both lines are closely interconnected: the higher the hardware development level is, the higher the intellectuality level can be achieved, and the automation level shall correspond to the technical level of promising high-efficiency power plants. Trends in the development of modern control systems based on the widespread implementation of software and hardware system (SHS) have provided for creation of the unified power unit control system in all modes of operation, that, in addition to direct control of the power unit, is capable of blocking operator mistakes, controlling intelligent regulation strategies, fully integrated power control and generation using digital process control, etc.

The analysis of the functional capabilities of modern APCS built on the basis of domestic and foreign SHS performed by the scientists of the National Research University "MPEI" shows that improving the process performance quality, optimizing their modes and parameters take place mainly at the equipment and power unit level and significantly to a lesser extent - at the power plant level [3-6]. The scientists of the Institute of Control Sciences of RAS have reached the same conclusion, and they state in [7] the following: "Unfortunately, the practice for APCS creation has developed "by prototype", the essence of which lies in the transfer of functions "prototype" to new hardware and software. In this case, it is clear that the APCS functional capabilities remain at the same level and, as a rule, are limited to the control of separate parameters and local control of separate production mechanisms. The author notes in the paper "Research and development of methods for automated control of processes and their configurations in flow-line production" [8]: "However, the analysis of many operating APCS (for example, APCS of site facilities) shows that today the APCS functionality is used at the same level - mostly SCADA (Supervisory Control and Data Acquisition) is actually the simple display of the process status. Currently, as a rule, only the parameters monitoring and local control of separate production facilities are automatically performed. At the same time, the coordination of processes and operations, interaction with related systems and services, process control as a whole and decision-making in extreme situations are still laid upon a person".

As a result, an insufficient level of automation and low technical and economic efficiency, as the costs required for actual hardware and software are difficult, and in some cases it is impossible to compensate for gains from the processes automation. Another conclusion from the performed analysis - when implementing control algorithms in the APCS based on the up-to-date SHS, their wide data and software capabilities are not fully used. Under these conditions, the problem is highly actual for development of methodological provisions, technical and process solutions, the implementation of which will ensure further increase in a degree of intelligence of energy production control systems at power plants up to a certain (optimum) level by developing and implementing new innovative technologies, algorithms and systems for optimum control of operation modes for power plants equipment and the power plants in general.

In order to search for possible ways to improve the intelligence of the SHS based control systems and to improve their efficiency, we carried out calculations to assess the technicaland-economic practicability of full-scale upgrade of 200 and $300 \mathrm{MW}$ condensing type power units APCS based on the domestic SHS "Kvint" provided that optimization tasks of all control levels are implemented based on SHS data support [9, 10]. The calculations have shown that if the entire economic effect from the introduction of the full-scale APCS with solving the block and plant level tasks are taken as 100 percent, then it is distributed among its components approximately as follows:

- Improvement of equipment process control quality and partial optimization of their parameters $-17.5 \%$; 
- Optimum control of equipment operating modes (including automated starts from different thermal states) $-17.5 \%$;

- By solving the problems for ensuring the optimum strategy of power unit application in the electricity and capacity market $-35.0 \%$.

- By increasing the reliability of the equipment functioning and reducing operating costs by optimizing the terms and scope of repairs and maintenance $-30.0 \%$.

The specified data show that the trends for development of intelligent automated process control systems (IAPCS) shall be aimed at creating the unified system for optimum control of technical and production processes in all modes of operation of equipment, power units and the plant as a whole.

The question arises - how to be out of the current APCS condition to a higher level in terms of intellectual and efficiency, i.e. how to implement the transition to the creation of a "smarter and more efficient" APCS. Is it possible to apply the basic principles of the Industry 4 to achieve this goal?

The term "Industry 4.0", as the next, the 4th stage of the industrial revolution, was meant as informatization of the assembly process and components move under supervision and machines interaction, but now this concept has more widely meaning and proposes rational use of natural and technical resources, the most effective energy saving based on maximum automation and intellectualization of production processes, control decisions, etc. [11, 12].

Although there is currently no clear algorithm for applying the concept of Industry 4.0 in various industries, its main principles are stated and can also be interpreted with regard to automated power generation at power plants [13]. One of the Industry 4.0 principles is the possibility of transition from the conventional vertical integration of production processes characterized by the centralization of production processes to the horizontal integration of various parts of the production process interacting with each other, while a significant part of production will be carried out automatically without human participation, i.e. the human role is reduced to the minimum. In the process of production control, an important place is given to the Internet of Things, i.e. the possibilities of using Internet information technologies for coordination and maintaining production processes.

The process of electric power generation at power plants in principle corresponds to the principle of processes interconnection between each other, but from the point of view of these processes control, if the APCS is built on the basis of SHS, a centralized control system is often applied, when all control decisions are made in the central server, where the whole information flows from measuring gages and instruments. For real, this does not apply to automatic control systems (ACS), which for the most part function in the autonomous mode. In this system, the process of production process optimization becomes more complicated due to the heavy workload of mid-level controllers that receive and process a huge amount of information.

The application of the horizontal principle of TPP processes control means the transition to geographically and functionally distributed control systems when a part of controllers is installed near the control facility and partly takes over the functions of data processing and processes controlling of this particular facility. The territorial distribution of the main generating units, auxiliary power facilities and technological assemblies is a compulsory necessity to ensure the vital activity of any large energy complex. The use of APCS local software systems supplied by manufacturers of completed main and auxiliary equipment has become a real necessity, but in this case, a certain complexity takes place in the installation of any complex geographically distributed system.

Nevertheless, the process of decentralization in the control process in the APCS built on the SHS is currently implemented during the design of new APCS and modernization of existing ones by setting the so-called peripheral, low-power controllers, but mainly for distant facilities. When applying the ideas of the Industry 4.0 such a control principle shall be 
extended to all the main units of the power unit, but in this case, it is necessary to ensure their interconnection in accordance with the process and with the operational control level. This will relieve the controllers at the level of operational control, especially in terms of data processing to solve common optimization tasks. In addition to purely technical and technological advantages - the improvement of operational and other conditions, it becomes possible to develop modern APCS in the line of their intellectualization by using highprecision mathematical models in the control circuit based on the typical diagnostic algorithms and optimum control.

The APCS design in accordance with this principle creates favorable conditions for the application of the basic concept Industry 4.0 - widespread implementation of IP technologies and the fullest automation of processes to increase the efficiency and intelligence of the production control system. The concept implementation with regard to the power plants SHS based APCS at current level of APCS, even if they are built on the SHS basis, is a complex problem due to the above mentioned, the application software ASW functions primarily to solve block control tasks, and the main economic effect of a full-scale APCS can be obtained when solving the plant level optimization tasks.

Taking into account this fact, the principle for building the control system to implement the concept Industry 4.0 can be suggested:

- Transition to control of technological and production processes of energy production at TPP by the criteria of energy and technical efficiency. Contrary to the proposals of separate solution of optimization tasks of unit, block and plant level existing in the technical literature leading in most cases to contradictory results, the problems solution in total will allow using the results obtained in the hierarchical approach "unit-block plant" at the lower hierarchies when solving the tasks at the upper levels; in this case, a clear separation of the solved optimization tasks shall take place at each hierarchical level approximately in the following form $[4,9]$ :

at the unit level - optimization of the parameters and indicators adjusted at the given unit (boiler efficiency, condenser pressure, steam pressure at sliding control of primary steam pressure, etc.); determination of the current technical condition (state coefficient) on the basis of parametric analysis (diagnostics);

at the block level - calculation and analysis of current and rated energy indicators (PI) of each unit and block as a whole, determination of the reasons for their deterioration and development of recommendations for their elimination; optimal distribution between parallel units operating included in the unit (double blocks, two, three-shaft CCP); operative and long-term diagnostics of unit equipment with optimization of terms of units repair and maintenance; optimum distribution of heat load between network heaters of the heating unit, etc.;

at the plant level - calculation and analysis of current and rated SHS of power units and the plant as a whole, taking into account the plant's own needs; selection of the composition of generating equipment, taking into account the possibilities of units back-up and optimal distribution of thermal and electrical load for SHS, and optimal distribution of electrical load for the condensing plant between generating units, taking into account the peculiarities of power plant output to the electricity and capacity market; choice of the optimal strategy for the scope and terms of repairs and maintenance, taking into account the current state of equipment with the development and implementation of automated systems for technical diagnosis, etc.

- Development and implementation of the concept of operational control of the power plant operating modes as part of the SHS application software in the automatic (or semiautomatic) mode, with the creation of tasks for the block and local APCS. In this case, the problem of the expediency for solving any optimization task at the given time, based on the current needs of the power plant. 
- In the framework of the above principle of interaction between the hierarchical levels of the distributed APCS, an algorithm shall be developed for optimal distribution of the APCS information support between peripheral, block and plant level controllers by the criterion of maximum speed.

An important aspect in the implementation of the principles of the Industry 4.0 in modern APCS is the current state of APCS hardware and a degree of their readiness for solving the above tasks.

Since the beginning of distributed microprocessor systems development, a certain possibility of their spatial distribution has been seen. With the development of microprocessor means and the accumulation of experience development, these opportunities have significantly expanded. Quite a large number of manufacturers of SHS with various own technical solutions has been appeared.

To implement the above tasks, the SHS shall meet certain requirements for hardware and software $[4,14]$. However, not all SHS meet these requirements. Thus, the network interface of remote modules can not be reserved, and the high-speed response of network interfaces in some SHS is not enough to implement protection and security locks, and controllers require forced ventilation that is not permissible when placed in contaminated industrial premises. Remote modules can often be installed only in the controller cells reduced in size that does not allow creating a truly distributed system.

Nevertheless, in Russia there are several domestic and imported SHS that fully meet the requirements for high-speed distributed systems of responsible control. The positive experience of field controllers operation is accumulated; typical solutions are developed for creation of local control systems on their basis. Several manufacturers serially produce drives smart control cabinets, intelligent sensor stands have been developed and begin to be used in projects. In this case, it can be stated that advanced domestic developments are as good as the characteristics and quality of products of the world's leading manufacturers.

The use of microprocessor technology along with SHS also affected field technical means of APCS: primary measuring instruments, shut-off and regulating actuators, local control devices and secondary indicating gages. There was such a thing as the intelligence of field control and monitoring hardware that allowed not only to increase the own hardware functionalities, but also to expand the APCS network architecture having included it to field equipment. A degree of intellectual security of the field level means that it performs the functions of preliminary processing of technological parameters, self-diagnostics, reconfiguration of measuring ranges and control in the on-line mode, parameter simulation. The use of switches - field bus protocol converters provides logical consolidation in not only a group of technological parameters into a single network node, but also technological functions of APCS.

Thus, when analyzing the effectiveness of development and implementation of the proposed APCS architecture and the structure of software and hardware of the TPP APCS, the following aspects shall be taken into account:

1. Installation of peripheral (field) controllers in the immediate vicinity of control objects and transferring the functions of primary data processing, partial control of local control systems (LCS), and a number of other functions to them. To reduce the data volume transferred to the upper level of data control and freeing CPU from solving particular problems, is accompanied under other equal conditions by reducing the reliability of the measurement systems as a whole.

2. The transition (partial or full) to the digital interface of data exchange between sensors and controllers using modern digital gages including intelligent sensors, actuators, etc. leads, on the one hand, to instruments cost increase, but, on the other hand, to disposal (partially or completely) of electromechanical communication devices and assemblies (when installing field semiconductor switches). 
3. Widespread introduction of field controllers, intelligent instruments and digital data transfer interface will lead to the need for a more clear-cut solution of the problems of redundancy and information security;

4. Extension of LCS application and functions increasing volume performed can lead to the possibility of a significant increase in the automation level and a volume of optimization tasks of the block and system nature to be solved at the upper level of control that will lead to increase in the APCS efficiency.

A special place in the process of SHS based APCS intellectualization is the problem of the level of technological and production processes automation. The current monitoring and control hardware allows automating rather complex processes. The most difficult processes are transient regimes: start, stop, load relief and extreme regulation modes. In addition, modern power plants operation in conditions of variable load schedules leads to the need for the operation of TPP power equipment in the modes of minimum safe, shutdown and subsequent start-up. Of course, these modes can be automated, but it requires a lot of material and intellectual costs.

In accordance with the above objectives and problems setting, a degree of automation of geographically distributed APCS is significantly increased, primarily, due to the solution of complex tasks. Nevertheless, at the block level this issue is solved in different ways. Most often, depending on the availability of material assets and misunderstanding of the long-term prospects for the energy complex development [14].

Conceptual provisions by a degree of energy facility automation shall be built under the conditions of "reasonable sufficiency" based on the theory and technological experience of maintaining the equipment operating modes.

According to the preliminary assessment, this approach can provide for the following: increase in power plants operation economy by optimizing the equipment operating modes within $2 \%$, decrease in fuel costs for declaration conditions by $20-30 \%$; - increase in power plants maneuverability by reducing the turbines start-up time by $40-50 \%$; increase by at least $40 \%$ of the reliability of equipment technical condition assessment; decrease in a number of emergency stops by $15 \%$; increase in the power equipment resource by at least $10 \%$.

APCS intellectualization by controlling the plant technical and production processes with applying the Industry 4.0 principles will require:

- Development and implementation of new approaches in the theory of automatic control of complex technical systems as applied to energy facilities when integrating the control circuits of processes of operational (at the equipment level) and station (pre-forecast) levels;

- Expansion of the SHS functional capabilities in terms of optimal control of technical and production processes at the block and station levels by integration of calculation algorithms of above tasks into the SHS software applications;

- Large computing power and powerful methodological and algorithmic base, necessary for calculations in the mode as close as possible to real time.

Below there is a brief description of the possible configuration of the proposed SHS based control system [15]. The system consists of mandatory and additional modules. The mandatory system modules are: calculation database, calculation server, data collection server. All these modules can be local, i.e. implemented on one computer. Such implementation will increase the system reliability and operation rate, but will reduce its scalability and significantly increase the computational load to a separate physical module. The system modularity will avoid large costs for its maintenance, allow for flexible configuration, provide interfaces to create the own modules for advanced users and thirdparty developers. Such implementation will increase the system reliability and operation rate, but will reduce its scalability and significantly increase the computational load to a separate physical module. The main requirement for the command control system of equipment 
operation modes is the presence of multiple $\mathrm{I} / \mathrm{O}$ interfaces for data exchange that requires special attention to information protection. For this purpose, it is necessary to provide the possibility for creation of a user with access rights to the built-in functions only. This will provide for testing the request before submitting the information. High network loading due to the applying the unified database requires the input of requests optimization algorithms. The calculation server is used for periodic or signal tasks start, which implies task program start. The data collection server performs the functions for data collection from the APCS and other systems and downloads the received information to the calculation database. For this module, it is necessary to provide multiple network communication protocols for access to other systems. Manual input, if necessary, can be performed using the clients' data collection server. The data transfer server is not a mandatory SHS module. In this case, its installation will significantly increase the system reliability. It serves to transfer operational information between the plant shift supervisor, power unit operators and other operational personnel in the automatic mode or on request. Data decoupling between different modules allows talking about the extensibility and interchangeability of system parts. In this case, data concentration in the unified database will prevent their duplication. Thus, all modules, both logical and physical, can be replaced, added or removed from the system.

The "archive" and "user interface" modules serve as additional ones. The main purpose of the archive is to back up the data. It is not a mandatory module, but in case of link broken it can serve as a backup storage and data provider for other modules. Such communication involves laying of redundant communication lines. In case of database server failure, all modules will change over the archive. When restoring the communication, the archive will start synchronizing the data with the calculation database.

User interface modules allow monitoring and controlling the software system behavior, making adjustments to the initial data, manual setting some parameters, adjusting the calculation results, sending tasks to the turbine engine operators' workstations, visualizing data in the form of graphs and tables.

\section{Conclusion}

Thus, the problem of increasing the energy efficiency of energy production at power plants can be solved by the intellectualization of the control system throughout the entire production chain of energy generation by applying the basic concepts of the "Industry 4.0" by implementing innovative, energy- and resource-saving technologies and algorithms for optimum control of technical and economic indicators, equipment operation modes when entering the station to the market of electricity and power, algorithms of implicit and longterm diagnostic of unit equipment with optimizing the terms and amounts of maintenance and repair.

The proposed principle for building the control system to implement the concept Industry 4.0 will increase the intellectual level of systems control and accelerate the creation of intelligent equipment and intelligent power plants

The developed methods will be effective in the design and optimization of processes and equipment, as well as building of high-quality power plants control systems. Using the methods and algorithms developed in the project will increase the intellectual level of the control systems and accelerate building of intelligent equipment and intelligent power plants.

\section{References}

1. Yu. S. Tverskoy, S. A. Talamanov, Teploenergetika. 10, (2010)

2. V.A. Bilenko, El. st., 1, 13-18 (2004) 
3. T. M. Arutyunyan, E. K. Arakelyan, V. A. Makarchian, Vestnik of MPEI, 6, 114-117 (2007)

4. E. K. Arakelyan, E. D. Vasiliev, S. R. Khurshudyan, Vestnik of MPEI, 1, 15-20 (2014)

5. E. K. Arakelyan, E.D. Vasiliev, Energosberezhenie I vodopodgotovka, 5 (97), 21-30 (2015)

6. G. Krokhin, A Pestunov, E Arakelyan, V Mukhin, IOP Conf. Series: Journal of Physics: Conf. Series 891, (2017)

7. I. V. Prangishvili, A. A. Ambartsumyan, A. G. Poletykin, G. G. Grebenyuk, I. B. Yadykin, State of energy facilities automation level and technical system solutions aimed at its improvement, Problems of Control (2003)

8. S. A. Branishtov Research and development of methods for automated control of processes and their configurations in flow-line production, diss. Ph.D. In science Moscow, 20 (2008)

9. E. K. Arakelyan, A. V. Andryushin, V. R. Sabanin, S.V. Mezin, F. F. Pashchenko, IOP Conf. Series: Journal of Physics: Conf. Series, 891, (2017)

10. E. K. Arakelyan, M. A. Panko, A. Sh. Aslanyan, Teploenergetika,(2010)

11. Katerina Bondar What is reality Industry 4.0 InnovaCima, 9 November, 2017 [online] Available at: http://www.en.unir.net (2018)

12. Bernar Marr What Everyone Must know about the Industry 4.0 Forbes June 20, 2016 [online], Available at: http://www.gitex.com (2018)

13. R. Rakhmanulov, Results of the conference "Industry 4", Control Engineering, Russia, August (2017)

14. V. S. Nevzgodin, V. A. Bilenko, A. G. Svidersky, P. A. Gorozhankin, Energy Reliability and Safety, 3, 19-27 (2011)

15. A. Chernyaev, Papers of International Scientific Seminar Networked embedded and control system technologies: European and Russian R\&D cooperation, Italy, Milan, (2009) 